Delft University of Technology

\title{
MPC Based Centralized Voltage and Reactive Power Control for Active Distribution Networks
}

Nguyen, Hoa Minh; Rueda, Jose L.; Lekic, Aleksandra; Pham, Hoan Van

DOI

10.1109/TEC.2021.3054844

Publication date

2021

Document Version

Accepted author manuscript

Published in

IEEE Transactions on Energy Conversion

\section{Citation (APA)}

Nguyen, H. M., Rueda, J. L., Lekic, A., \& Pham, H. V. (2021). MPC Based Centralized Voltage and Reactive Power Control for Active Distribution Networks. IEEE Transactions on Energy Conversion, 36(2), 15371547. [9340314]. https://doi.org/10.1109/TEC.2021.3054844

\section{Important note}

To cite this publication, please use the final published version (if applicable).

Please check the document version above.

\section{Copyright}

Other than for strictly personal use, it is not permitted to download, forward or distribute the text or part of it, without the consent of the author(s) and/or copyright holder(s), unless the work is under an open content license such as Creative Commons.

\section{Takedown policy}

Please contact us and provide details if you believe this document breaches copyrights.

We will remove access to the work immediately and investigate your claim. 


\title{
MPC Based Centralized Voltage and Reactive Power Control for Active Distribution Networks
}

\author{
Hoa M. Nguyen, J.L. Rueda Torres, Senior Member, IEEE, Aleksandra Lekić, Member, IEEE, \\ and Hoan V. Pham
}

\begin{abstract}
The paper presents an approach for online centralized control in active distribution networks. It combines a proportional integral (PI) control unit with a corrective control unit (CCU), based on the principle of Model Predictive Control (MPC). The proposed controller is designed to accommodate the increasing penetration of distributed generation in active distribution networks. It helps in continuously satisfying the reactive power requirements of the transmission system operators (TSOs), while maintaining an acceptable voltage profile in the active distribution network, and simultaneously minimizing the total active power losses. The controller also ensures compliance to operation requirements of distribution network operators (DNOs). By replacing the full load flow (LF) calculation with sensitivities, derived from a linearized model of the network, the controller can work in real-time applications. Moreover, the computational burden of the proposed controller is reduced since the $\mathrm{CCU}$ is activated only when a voltage violation or considerable change of operation condition occurs. The performance of the proposed controller is demonstrated on a 11-kV test network with 75 buses and 22 distributed generators.
\end{abstract}

Index Terms - Voltage control, distributed generation, reactive power management, model predictive control, artificial neural networks, smart grids.

\section{INTRODUCTION}

$\mathrm{I}^{\mathrm{s}}$ NTERMITTENT renewable energy resources (RES), like solar and wind, will be massively connected to European distribution networks due to very high levels of renewable energy sources (RES). RES will reach $75 \%$ of the total demand in 2040 [1]. This entails several challenges as system reliability, security and optimal operation, especially in the short term operation time frame, where a high degree of variability of power supply from RES may occur [2-3]. Besides, transmission systems in Europe are increasing in complexity. E.g. reactive power reserves, available in the transmission system, are diminishing as mainly converter connected RES replaces synchronous connected generation. Consequently, it is necessary to ensure the development of robust approaches to ensure that voltage excursions stay within permissible limits.

This work is supported by the research foundation of Tra Vinh University, Vietnam. (Corresponding author: Hoan V. Pham).

Hoan V. Pham and Hoa M. Nguyen are with the Department of Electrical Engineering, Tra Vinh University, Vietnam (e-mail: pham.v.hoan@tvu.edu.vn; hoatvu@tvu.edu.vn).

J.L. Rueda Torres and A. Lekić are with the Department of Electrical Sustainable Energy, Delft University of Technology, Delft, the Netherlands (email: J.L.RuedaTorres@tudelft.nl; A.Lekic@ @udelft.nl).
Thus, the European Commission (EC) defines a set of common requirements for an interface between transmission and distribution networks. EC also requests that DNOs comply with a contractual PQ diagram [4]. For instance, transmission connected distribution grids in Switzerland face payments if the power factor of exchanged (imported and exported) power is outside 0.9 inductive to 0.9 capacitive [5]. Furthermore, the need for increased cooperation between system operators is widely recognized, especially in a scenario with increasing renewable energy sources (RES) and increasing participation of DER to ancillary services markets. For instance, there was a shift in preference from passive control schemes to 'active network management' (smart grid) [3],[6]. A smart grid increases the level of coordination because system operators can support each other in the efficient and cost-effective operation.

Several studies have investigated the reactive power exchange at the transmission-distribution interface, but, in most of the cases, they were focused on the problem only from the DNO's point of view, considering stiff voltages in the TSO grid and pre-defined ranges of reactive power exchange. In [7], a two-stage optimization routine is developed to tune controllers such that the power confidence levels at the HV/MV interface remain within specified limits. In [8], the author proposes two-time scales to schedule the planning and to perform the operational optimization of interface powers considering uncertainties. The authors in [9]-[11] propose different measures to reduce impact of DGs on the transmission system by regulating the reactive power exchange at the point of common coupling (PCC). Similarly, a mean-variance mapping optimization (MVMO)-based controller for an online optimal control of reactive power sources in wind farms is proposed in [12] to fulfill the grid requirements at PCC. An active network management approach is proposed in [13] to limit the reactive power export to National Grid and it is based on a multi-period optimal power flow framework. A coordination mechanism between TSO and DNO based on an Optimal Power Flow (OPF) tool featuring Model Predictive Control (MPC) and MultiObjective (MO) optimization is presented in [14]. The work presented in [15] provides the fundamentals for an MPC application for voltage control in active distribution networks. In [16] an MPC controller has been presented in order to control both the voltages in distribution systems and the reactive power exchanged at the TSO-DNO interface by maintaining the ratio of reactive power over active power, consumed by the MV network, inside a range of predefined values. Besides, to ensure that the voltage set-point given by TSO always leads to feasible DNO operation, the authors in 
[17]-[19] proposed different approaches for incorporating information between TSO and DNO. More recent works introduce concepts of active, reactive power, and voltage controls, taking into account different scenarios. The work presented in [20] employs discretized state-space equations to represent system's operation. Similarly, in [21], the optimal power flow formulation of the MPC is provided. However, both methods [20]-[21] require a detailed formulation of the mathematical equations, which cannot be intuitively defined. In order to speed up control, in [22], it is proposed to use a decoupled MPC, where OLTC tap changes are characterized with slow dynamics, and DGs active and reactive power changes with fast dynamics.

The main objective of this paper is to introduce a MPCbased centralized controller for an online optimal control of reactive power sources in distribution networks. Beyond the current state-of-the-art on MPC applications for this purpose, this paper proposes a new centralized controller for the online active distribution network management. Particularly, the controller is able to optimally drive the network by using measurements from selected buses, subjected to operational security constraints (i.e. no violation of voltage limits). In comparison with the most of the published algorithms, the proposed variant of MPC uses sensitivities estimated through artificial neural network (ANN), and, thus, it does not require extensive mathematical calculations. The architecture of the controller comprises the two following controllers:

- A PI controller with a slow response, which undertakes a task of fulfilling requirements of TSO (i.e. reactive power import/export from distribution networks at the PCC). This is done by equally distributing reactive power demand into available VAr sources.

- A controller, based on the MPC principles and the calculation of sensitivities, which, due to the reduced computational burden, enables its application in real-time operation. The controller helps in satisfying DNO's requirements, such as stable voltage profile within pre-defined technical bounds, and the minimum losses. The controller serves as a corrective control unit (CCU). Remarkably, it is only trigged when necessary, to correct control actions in

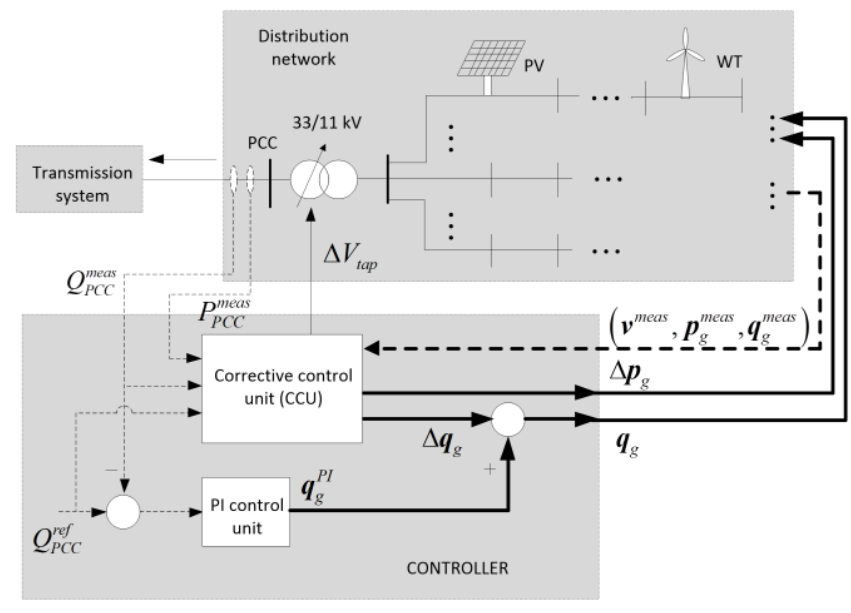

Fig. 1. The proposed controller scheme. optimal manner based on a small set of selected measurements and an offline trained model.

The paper is organized as follows. Section II explains the overall operation principle of the proposed controller. Section III provides a detailed description of the CCU. The test network and algorithm implementation together with the simulation results are given in Section IV. Finally, concluding remarks are summarized in Section V.

\section{Proposed Control Strategy}

The proposed controller's scheme is sketched in Fig. 1. The controller consists of two main control units: the PI control unit and the CCU inspired by MPC. While the PI control unit carries out fulfilling reactive power demand $\left(Q_{P C C}^{r e f}\right)$ requested by TSOs (e.g. to meet certain grid code requirements), the $\mathrm{CCU}$ corrects the control variables' values in order to minimize losses and correct voltages. The controller presented in this paper focuses on operations characterized by a slow time response (e.g. 10 seconds to few minutes) to adapt the DG response to changing steady-state requirements. It is emphasized that the proposed controller is exclusively conceived (from DNO's point of view) for continuous fulfillment (in an optimal manner) of grid code requirement at PCC, and not for system-wide reactive power control purposes. Thus, an analysis from a large-scale system performance perspective falls outside the scope of this paper. In this study, there are three types of control variables [15]:

$$
\boldsymbol{u}=\left[\boldsymbol{q}_{g}^{T}, V_{\text {tap }}^{T}, \boldsymbol{p}_{g}^{T}\right]^{T}
$$

where ${ }^{T}$ denotes vector transpose. Vectors $\boldsymbol{p}_{g}$ and $\boldsymbol{q}_{g}$ are active and reactive power injections of DGs, while $V_{\text {tap }}$ is a voltage set-point of the transformer.

The action of the control algorithm shown in Fig. 1 is summarized in following steps:

- The proposed controller collects necessary measurements, such as $Q_{P C C}^{\text {meas }}, \boldsymbol{v}^{\text {meas }}, \boldsymbol{p}_{g}^{\text {meas }}$, and $\boldsymbol{q}_{g}^{\text {meas }}$. Where $Q_{P C C}$ and $\boldsymbol{v}$ are the reactive power exchanged at PCC and the measured voltages at selected buses, respectively. The subscript 'meas' stands for measurements. It should be noted that the actual losses and the energy that "leaves" the distribution network are usually quantified in practice. The difference between the aggregated consumption data, provided from the settlement system, and the GSP measured energy, represents the overall network losses. The maximum sampling time for real-time applications is $1 \mathrm{~s} \mathrm{[23].}$

- Next, the controller provides reactive power set-points of the DGs $\left(\boldsymbol{q}_{g}=\boldsymbol{q}_{g}^{P I}+\Delta \boldsymbol{q}_{g}\right)$. Where $\boldsymbol{q}_{g}^{P I}$ is the reactive power injection by the DGs requested by the PI control unit, $\Delta \boldsymbol{q}_{g}$ refers to the change of DGs reactive power. The change of the OLTC transformers' voltage set-point is $\Delta V_{\text {tap }}$, and the curtailment of the active power injection of DGs is $\Delta \boldsymbol{p}_{g}$. These control actions are sent to the corresponding components across the network. 
- Except for the reactive power $\boldsymbol{q}_{g}$, which is calculated in the controller at local level, other set-points $\left(V_{t a p}, \boldsymbol{p}_{g}\right)$ are computed as illustrated in Fig. 2. The value $V_{\text {tap }}^{\text {ref }}$ is the reference set-point of the tap changer, whereas $\boldsymbol{p}_{g}^{\text {ref }}$ is calculated based on the actual operating conditions (e.g. influenced by the actual wind speed, solar intensity). Note that the subscript ' $r e f$ ' stands for reference value.

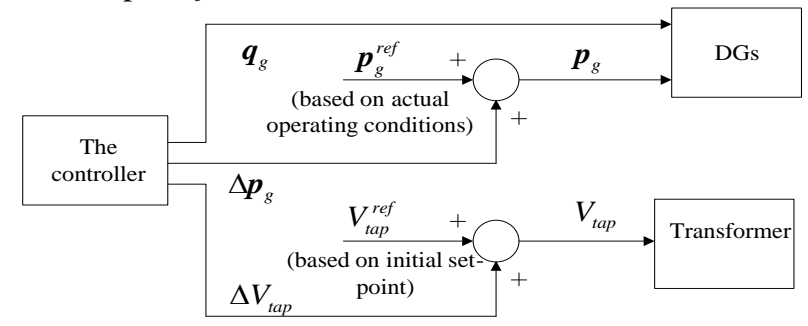

Fig. 2. Calculation of set-points at local level.

\section{The CORRECTIVE CONTROL UNIT}

The CCU, depicted in Fig. 3, is triggered occasionally, under predefined conditions (voltage violation, operation condition change), to correct voltage profile and to minimize the total losses. When it is triggered, the CCU starts collecting available real-time measurements. Some of the measurements are used as an input of an artificial neural network (ANN). The ANN is trained offline to output sensitivities. The process of determining the control actions is performed based on the online measurements and the sensitivities provided by the ANN. To prevent inaccurate measurements, the new measurements for the next calculation iteration are performed after a time delay $T_{\text {delay }} . N_{\text {step }}$ is a predefined value which limits the number of $\mathrm{CCU}$ iterations per triggering. Hence, $N_{\text {step }}$ defines a MPC horizon.

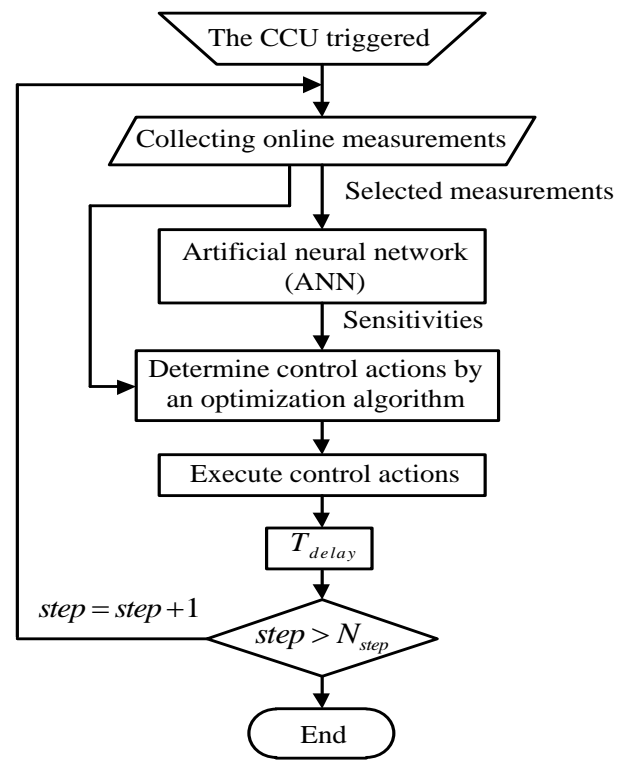

Fig. 3. Flowchart of the CCU operation principle.

\section{A. Conditions for triggering the $C C U$}

To reduce the computation burden, the CCU is only triggered if one of the following conditions occur:
- Voltage violation.

- Predefined change level of operation condition: When the networks significantly change their operation condition (e.g. load profile variation identified from day to night or reactive power requirement of TSO corresponding to a predefined step change), the networks' optimal operation setting-points need to be adjusted accordingly. In this study, it was assumed that the step change of 2 MVAR corresponding to reactive power capacity of a DG will trigger the CCU.

\section{B. Artificial Neural Network}

As indicated earlier, the ANN is used to approximate sensitivities. The ANN is trained by using several online measurements as its inputs. The procedure for creating the ANN involves several steps, as shown in Fig. 4. The ANN training process is performed offline. Firstly, a database is created by using past, predicted, or approximated load profiles. A classification of the input data of the ANN, as well as the calculation of sensitivities (considered as the output), from the created database is performed afterwards. Next, the architecture of the ANN is defined according to the set of input and output data. The process of training is carried out to specify parameters of the ANN which are stored afterwards.

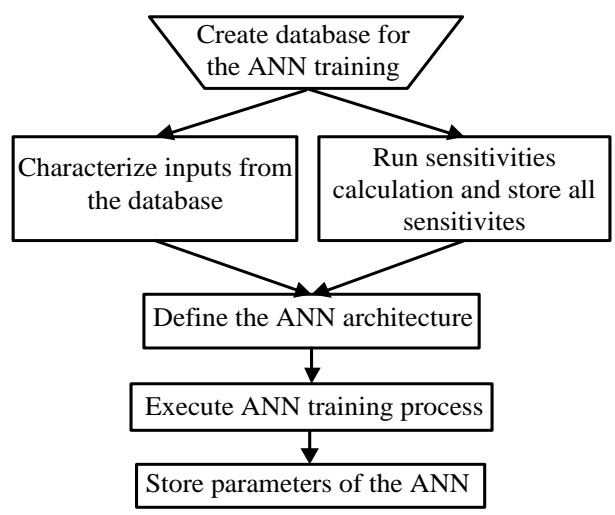

Fig. 4. Flowchart of building the ANN.

In this study, a multi-layer feedforward network based on backpropagation learning procedure is designed. It approximates sensitivities of the active power losses. The size of the input and output layers are defined by the measurements and the number of sensitivities, respectively. The selected neural network architecture consists of one input layer of logsigmoid neurons, followed by an output layer of linear neurons, and it is trained by using the functions of the MATLAB Neural Network Toolbox. The example proposed in the paper is tested by using two scenarios with different numbers of input and output layers. It is worth recalling that the CCU requires the ANN to provide the sensitivities of the active power losses with the respect to reactive power injections $S_{q_{g}}^{P_{\text {loss }}}=\frac{\partial \boldsymbol{P}_{\text {loss }}}{\partial \boldsymbol{q}_{g}}$, where $\boldsymbol{P}_{\text {loss }}$ is a vector of the active power losses and $\boldsymbol{q}_{g}$ is a vector of DGs' reactive powers. 


\section{CCU operation principles}

1) Calculation of Sensitivities at every operating point

The calculation of sensitivities is based on two methods. A direct method uses the steady-state power equations of load flow calculation. For example, the inverse of the Jacobian matrix provides the sensitivity of bus voltages with respect to the power variations. The equations presented in [24] can be used to compute the sensitivities between different variables. Another method approximates sensitivities based on load flow calculations, each one obtained by a small perturbation (e.g. $10 \%$ of the nominal value, as used in this paper) of a control variable. In this study, the latter is used to calculate the sensitivities of the control variables with the respect to the state variables.

\section{Determination of the Control Actions}

The MPC CCU calculates the change of control variables $\Delta \boldsymbol{u}(k)$ at a time instant $k$, as shown in (2), not only to maintain the monitored voltages within permissible limits, but also to minimize the active power losses.

$$
\Delta \boldsymbol{u}(k)=\left[\Delta \boldsymbol{q}_{g}(k)^{T}, \Delta V_{t a p}(k)^{T}, \Delta \boldsymbol{p}_{g}(k)^{T}\right]^{T}
$$

with $\Delta \boldsymbol{u}(k)=\boldsymbol{u}(k)-\boldsymbol{u}(k-1)$.

\section{1) Formulation of the Overall Objective Function}

The overall objective function of the CCU is a function of multiple objectives associated with the following control variables.

\section{a) Reactive Power of DGs}

Different objective functions can be used by the system operators. Besides the traditional minimization of the active power losses, there are other approaches such as the minimization of reactive power cost [25], [26], minimization of the deviations from contracted transactions [27], or minimization of the cost of adjusting reactive power control devices [28]. In this study, the minimization of the active power losses is selected, and the objective function (OF) at time instant $k$ is therefore defined as follows:

$$
O F_{1}(k)=\min \left(P_{\text {loss }}^{\max }+\sum_{i=1}^{N_{g}} S_{q_{g i}}^{P_{\text {loss }}} \Delta q_{g i}(k)\right)
$$

where $P_{\text {loss }}^{\max }$ is maximum active power losses over the distribution network, assumed to be equal to $10 \%$ of total loads in this study. As already mentioned $S_{q_{g i}}^{P_{\text {loss }}}$ represents sensitivity of the losses power with the respect to the $\mathrm{j}$-th component of reactive powers vector.

\section{b) Tap-changer of the Transformer}

The higher number of OLTC operations is proportional to higher cost in maintenance and reflects a reduction of the lifetime. Consequently, the minimization of tap changer operations is often a adopted as an optimization target. On the other hand, tap changers will be activated when: i) Changes in operation conditions push the controlled voltage at substation $V_{c t r}$ out of the dead-band; and ii) A shift of the dead-band is caused by a change of $V_{\text {tap }}$ when the controlled voltage at a substation $V_{c t r}$ is out of the dead-band. Therefore, the minimization of the change of $V_{\text {tap }}$ could lead to the least number of OLTC movements. The corresponding objective function is therefore defined as

$$
O F_{2}(k)=\min \left(\left|\Delta V_{\text {tap }}(k)\right|\right)
$$

c) Active Power Generation of DGs

The maximization of the active power production from DGs entails an economical advantage. This is permanently satisfied at local level because DGs always try to capture the maximum power by tracking actual conditions, such as wind speed, solar. However, under certain operation conditions, such as voltage violation, some curtailments of active power of DGs are needed. Consequently, the minimization of the active power curtailment becomes necessary to safeguard the network operation. Hence, the objective function is defined as

$$
O F_{3}(k)=\min \left(\sum_{j=1}^{N_{g}}\left|\Delta p_{g j}(k)\right|\right)
$$

where $N_{g}$ is the number of DGs.

The CCU is essentially an MPC which finds a sequence of control actions in $N_{C}$ steps (MPC horizon). Therefore, the overall objective of the CCU is formulated as a composition of the objective functions defined by (3)-(5), under a relation described by a weighting matrix $\boldsymbol{w}$, to penalize expensive control actions. In short, the composite problem is defined as

$$
O F(k)=\min \sum_{i=0}^{N_{C}-1}\left(\sum_{j=1}^{3} w_{j} O F_{j}(k+i)\right)
$$

subject to:

- Constraints of control variables

$$
\boldsymbol{u}^{\min } \leq \boldsymbol{u}(k+i) \leq \boldsymbol{u}^{\max }
$$

- Constraints of voltage

$$
\boldsymbol{V}^{\min } \leq \boldsymbol{V}(k+i) \leq \boldsymbol{V}^{\max }
$$

- Constraint of reactive power exchange with external grid

$$
\left|Q_{P C C}^{\text {ref }}-Q_{P C C}^{\text {meas }}(k+i)\right| \leq \varepsilon
$$

Equation $(7 \mathrm{c})$ indicates that control actions of the CCU do not cause any interaction with PI control unit. Therefore, $(7 \mathrm{c})$ can be translated into

$$
\left|\left(\nabla_{u} Q_{P C C}\right) \Delta \boldsymbol{u}(k+i)\right| \leq \varepsilon
$$

where $\nabla_{u}$ represents gradient of the vector of control variables $\boldsymbol{u}$.

The weighting matrix $\boldsymbol{w}$ is intuitively chosen in such way that the effects of the reactive powers have the biggest influence on the objective function and that active powers have the smallest effect. It ensures the highest priority to reactive powers (OF1). If the constraints are still not fulfilled, variables of OF2 (tap voltage) will be used. After that, if they are still not satisfied, variables of OF3 (DGs active powers) will be used as the last resort. Therefore, tuning the weighting matrices in this paper is merely done by choosing the weighting factor for OF2 much bigger than for OF1 and much smaller than for OF3.

2) The Voltage Constraint - Problem and Adaptation into the Optimization Algorithm

The constraint (7b) can be rewritten as follows: 


$$
\boldsymbol{V}^{\text {min }} \leq \boldsymbol{v}^{\text {meas }}(k \mid k)+\sum_{l=0}^{i} \Delta \boldsymbol{V}(k+l) \leq \boldsymbol{V}^{\max }
$$

then, it can be transformed into:

$$
\boldsymbol{L B}(k+i \mid k) \leq \sum_{l=0}^{i} \Delta \boldsymbol{V}(k+l) \leq \boldsymbol{U} \boldsymbol{B}(k+i \mid k)
$$

with $\left\{\boldsymbol{L} \boldsymbol{B}(k+i \mid k)=\boldsymbol{V}^{\text {min }}-\boldsymbol{v}^{\text {meas }}(k \mid k)\right.$ : Lower bound

$$
\boldsymbol{U} \boldsymbol{B}(k+i \mid k)=\boldsymbol{V}^{\text {max }}-\boldsymbol{v}^{\text {meas }}(k \mid k) \text { : Upper bound }
$$

and $\Delta \boldsymbol{V}(k+l)$ is calculated based on the impacts of the three following events:

$$
\Delta \boldsymbol{V}(k+l)=\Delta \boldsymbol{V}_{1}(k+l)+\Delta \boldsymbol{V}_{2}(k+l)+\Delta \boldsymbol{V}_{3}(k+l)
$$

- $\Delta \boldsymbol{V}_{1}$ are impacts of control actions on voltage changes.

$$
\Delta \boldsymbol{V}_{1}(k+l)=\left(\nabla_{\boldsymbol{u}} \boldsymbol{V}\right) \Delta \boldsymbol{u}(k+l)
$$

- $\Delta \boldsymbol{V}_{2}$ is treated as known disturbances due to slow response of tap position changes.

$$
\Delta \boldsymbol{V}_{2}(k+l)=\frac{\partial \boldsymbol{V}}{\partial V_{\text {tap }}} \Delta V_{c t r}^{\text {tap }} \gamma(k+l)
$$

- $\quad \Delta \boldsymbol{V}_{3}$ appears in a case that can occur at the time of measurement collection by CCU, if the PI control unit does not fulfill reactive power exchange due to its slow response. Consequently, the $\mathrm{CCU}$ has to account for the influence on voltages caused by further output evolution of PI control unit as follows:

$$
\begin{gathered}
\Delta \boldsymbol{V}_{3}(k+l)=\left(\nabla_{\boldsymbol{q}_{g}} \boldsymbol{V}\right) \Delta \boldsymbol{q}_{g}^{P I}(k+l) \\
\Delta \boldsymbol{q}_{g}^{P I}(k+l)=\left(\frac{Q_{P C C}^{r e f}(k+l)-Q_{P C C}^{\text {meas }}(k+l)}{N_{g}}\right) \cdot\left(S_{q_{g}}^{Q_{P C C}}\right)^{-1}
\end{gathered}
$$

where $\Delta \boldsymbol{q}_{g}^{P I}$ is reactive power of DGs further required by PI controller, and $\nabla_{\boldsymbol{q}_{g}} \boldsymbol{V}$ is sub-matrix of the matrix $\nabla_{\boldsymbol{u}} \boldsymbol{V}$. Equation (14) is determined with the consideration that reactive power at PCC is controlled using one PI controller. It is assumed that all DGs are influencing this error. Thus, this PI control produces a change at every DG, and the error is divided by the number of DGs, and by multiplying it by the corresponding sensitivity.

On the other hand, to avoid excessive control actions caused by inaccurate sensitivities leading to voltage violation, and to ensure voltages within their limits at the end of the prediction horizon, the range of voltage bounds should be narrowed at the beginning of the control horizon, and progressively expanded to its actual value up to the end of control horizon. Consequently, the voltage bounds in (9) can be adaptively adjusted as follows:

$$
\left\{\begin{array}{l}
\boldsymbol{L B}(k+i \mid k)=\lambda(k+i) \boldsymbol{L B}(k+i \mid k) \\
\boldsymbol{U B}(k+i \mid k)=\lambda(k+i) \boldsymbol{U B}(k+i \mid k)
\end{array}\right.
$$

with $\lambda(k+i)=\frac{N_{C}-i}{N_{C}\left(N_{C}+1\right)}$.

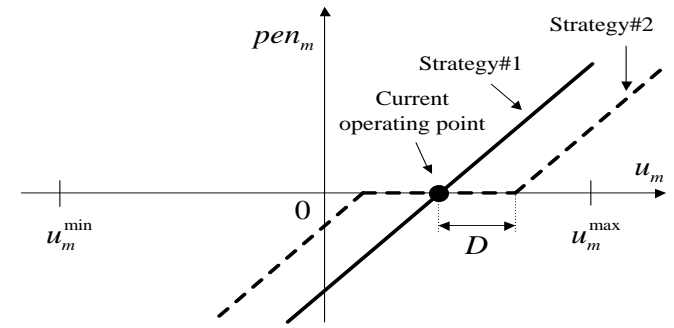

Fig. 5. Determination of penalty value of each control variable $u_{m}$.

3) Modification of the Overall Objective Function to Achieve Better Performance

In this paper, the sensitivities are calculated based on a small perturbation of each control variable (e.g. smaller than $10 \%$ of its nominal value). Therefore, the modification of the overall objective, by changing the magnitude of control variables around their actual values, is necessary to enhance accuracy of the sensitivities. To do so, an additional penalty term imposed on the change of control variables should be added in the objective function. Therefore, (7) is changed as follows:

$O F(k)=\min \sum_{i=0}^{N_{C}-1}\left(\sum_{j=1}^{3} w_{j} O F_{j}(k+i)+\sum_{m=1}^{N_{g}} \operatorname{pen}_{m}(k+i)\right)$

where pen $_{m}$ can be calculated using two different strategies, as presented in Fig. 5. In the Strategy\#1 penalties are estimated as

$$
\operatorname{pen}_{m}(k+i)=\alpha \Delta u_{m}(k+i)
$$

where positive real number $\alpha$ is a slope. In the Strategy\#2 a dead-band is used to penalize only large change of control variables. Thus,

$$
\operatorname{pen}_{m}(k+i)=\left\{\begin{array}{l}
0, \text { if }\left|\Delta u_{m}(k+i)\right| \leq D_{m}, \\
\alpha \Delta u_{m}(\mathrm{k}+\mathrm{i}), \text { otherwise }
\end{array}\right.
$$

The introduction of $D_{m}$ indicates that only the change of $m$-th control variable is encouraged. The value of $D_{m}$ should be selected equal to the value of perturbation of these variables in sensitivities calculation process. Hence $D_{m} \leq 10 \%$ of the nominal value.

It should be noted that the MPC represents a feasible problem. The selected objective function covers a convergent system if it is satisfied that for the objective function

$$
\begin{gathered}
l(\boldsymbol{u})=\sum_{i=1}^{N-1}\left(w_{1} P_{\text {loss }}^{\max }+w_{1} \sum_{j=1}^{N_{g}} S_{q_{g j}}^{P_{\text {las }}} \Delta q_{g j}(k+i)+w_{2}\left|\Delta V_{\text {tap }}(k+i)\right|+\right. \\
\left.+w_{3} \sum_{j=1}^{N_{g}}\left|\Delta p_{g j}(k+i)\right|+\sum_{m=1}^{N_{g}} \operatorname{pen}_{m}(k+i)\right),
\end{gathered}
$$

which is always positive, it is satisfied that $V_{N}^{0}(\boldsymbol{u}(k))=\sum_{i=1}^{N_{C}-1} l(\boldsymbol{u}(k)) \geq 0 \quad$ and $\quad$ that $\quad$ stems inequality $V_{N}^{0}\left(f\left(\kappa_{N}(\boldsymbol{u})\right)\right)-V_{N}^{0}(\boldsymbol{u})+l\left(\kappa_{N}(\boldsymbol{u})\right) \leq 0$. The Lyapunov control function $V_{N}^{0}(\boldsymbol{u}(k))$ is always positive, since it presents a sum of positive functions over the prediction horizon $N_{C}$. It presents a Lyapunov control function because it satisfies definition 2.31 on the page 126 in [29]. Outside of the 
prediction horizon $\Delta \boldsymbol{u}(k)$ is zero, and thus, $\boldsymbol{\kappa}_{N}(\boldsymbol{u})$ is zero. It can be concluded that $V_{N}^{0}\left(f\left(\kappa_{N}(\boldsymbol{u})\right)\right)-V_{N}^{0}(\boldsymbol{u})+l\left(\kappa_{N}(\boldsymbol{u})\right)=-V_{N}^{0}(\boldsymbol{u}) \leq 0$, which proves the stability.

Regarding the polytopic nature of the constraints, the system is convex if on the polytope facets the Lyapunov function "drags" the system into origin [30], see Fig. 6 where the origin (or a steady-state, or also called operating point) is denoted as 0 . There is well established theory how to tune such a system and we can refer to the work of Yuzo Ohta [31, 32]. With this approach state variables operate inside "socalled" polytope (see Fig. 6) and the control using Lyapunov function should ensure that the system stays inside the polytope, or that from any point outside it, it converges towards origin. The idea for tuning the weights in this approach relies on constructing negative time derivative of the piecewise-linear Lyapunov function, which should be orthogonal to the side of polytope or so-called facet, see orthogonal vector $\mathbf{f}_{1}$ on Fig. 6 . Multiple algorithms have been design for it, see [31, 32]. The mentioned approach provides fundament on how to define convex polyhedron (polytope) and on that way gives an estimate of the weights used inside piecewise-linear Lyapunov function, because they are equation related. This is possible because the Lyapunov function is directly related to the polytope and they are both using the same weights.

Since, for the detailed convexity analysis it is needed to check system equations at the each facet and Filippov criteria on the interconnection of influence of two constraints, the proof is omitted in this paper. However, for the each simulated case, the convexity of the problem is visible from its convergence into the steady-state.

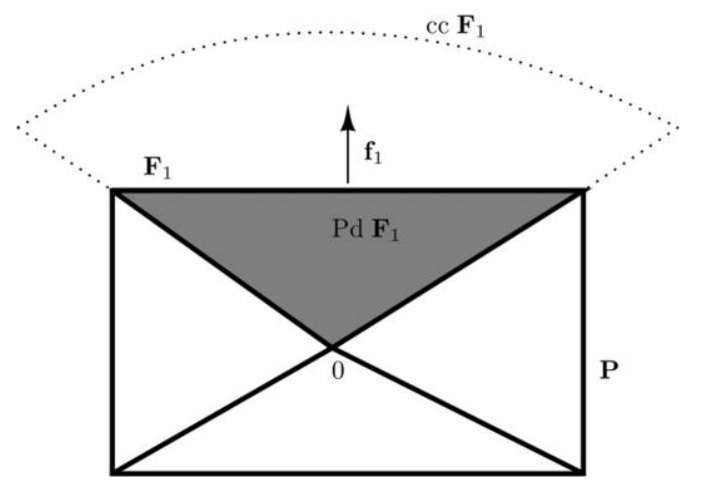

Fig. 6. Polytope example.

\section{E. Adaptive Selection of the Control Horizon}

The minimization of active power losses, when the controller works in loss control mode, is the primary task during normal operation. To reach this target, the controller must ensure acceptable voltages, even when subjected to inaccurate sensitivities. This can be achieved by setting $N_{C} \geq 2$, which however leads to more computational burden.

By contrast, when a voltage violation takes place somewhere over the network, the voltage control mode should be activated. It is expected that the voltage returns within the limits as fast as possible. Hence, $N_{C}$ should be minimal (or $N_{C}=1$ ).

\section{ALGORITHM IMPLEMENTATION AND RESULTS}

\section{A. Test Network and Measurement Deployment}

The test network depicted in Fig. 7 is taken from an U.K. generic distribution network (UKGDN), available in [33]. It comprises of 75 load buses (except bus \#1000 and bus \#1100) and 22 DG units (3 MW in the nominal capacity of each). It connects to transmission system through a cable (Thévenin reactance of 0.1 p.u of 200 MVA short-circuit power) and a $33 / 11 \mathrm{kV}$ transformer equipped with OLTC which is able to regulate voltage in range of $\pm 10 \%$ nominal voltage at distribution network side with 19 tap positions. The reactive power capacity of each DG is limited in range \pm 2 MVAR.

Besides, measurements at all DG buses, voltages of several additional buses are also measured as in Fig. 7. It is expected that if voltages at measured buses are admissible, voltages at the other busses are also admissible.

In the following subsections all numerical experiments were performed on a HP Pavilion Laptop with an Intel ${ }^{\circledR}$ Core $^{\mathrm{TM}}$ i7 5500 central processing unit (CPU), $2.4 \mathrm{GHz}$ processing speed and $8 \mathrm{~GB}$ random access memory.

\section{B. Characteristics and Parameters Setup of the Controller:}

\section{1) The ANN}

Active power loads in the normalized half-hour span and generation profiles over one year are provided in the UKGDN. Load profiles are given in form of consumer class that can be Domestic/Unrestricted (D/U), Domestic-Economy (D/E), Industrial (I), and Commercial (C). The methodology, given in [34] and approximation methods of mixing consumer classes at each bus to create load profile (used as the database of the ANN) are adopted in this paper. The ANN training takes database consisting of a whole year with 17520 half-hour time steps.

\section{2) Parameters Setup and Algorithm Selection for the Controller}

The aim of the controller is to preserve voltages in the distribution network within $\pm 5 \%$ of their nominal values. Table I presents controller parameters. The parameters of the

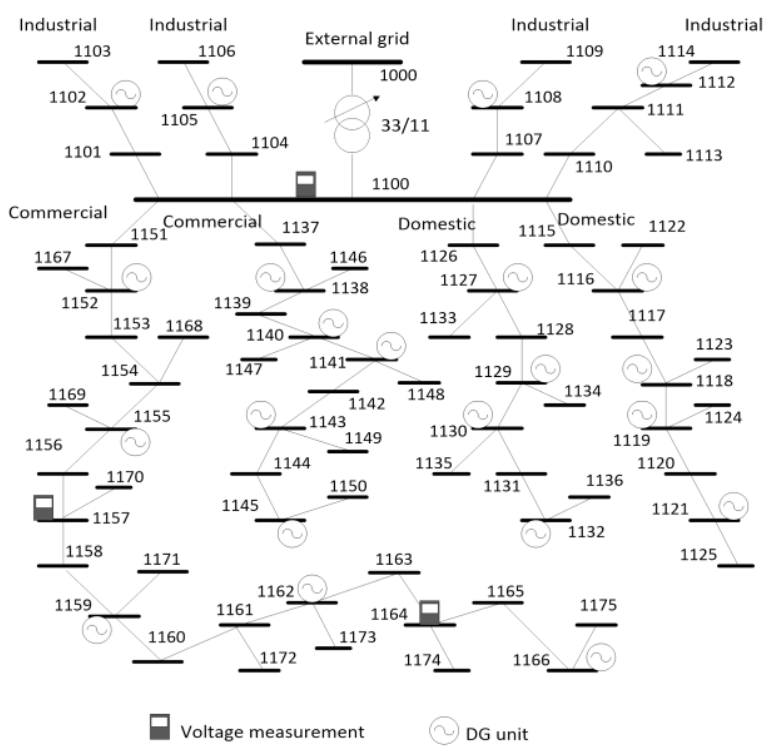

Fig. 7. Test network. 


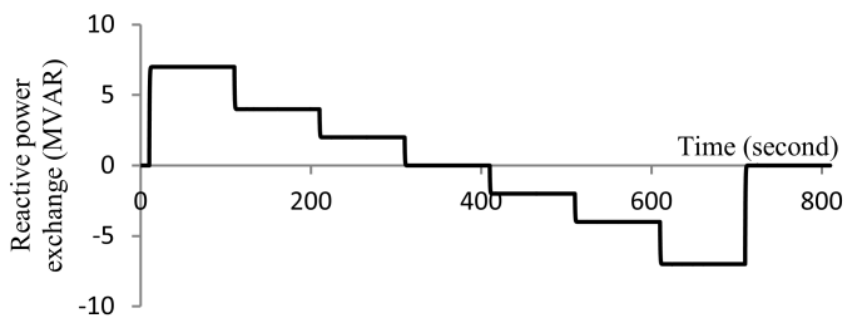

Fig. 8. Correction of reactive power exchange.

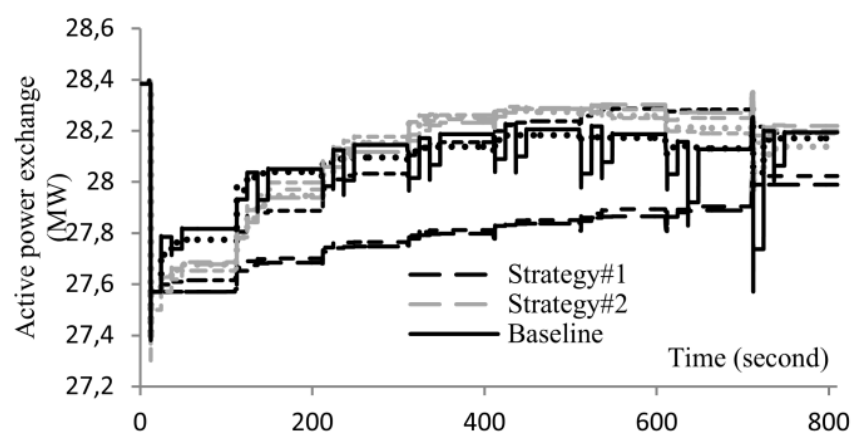

Fig. 9. Active power exchange inversely proportional to losses reduction: Largely to small dashed black lines describe Strategy\#1 with $\alpha$ from bigger to smaller. The similarly featured lines but with light black color present Strategy\#2, and solid black line is of Baseline.

PI controller are selected to ensure slow reaction (e.g., within a time frame of around $10 \mathrm{~s}$ to a few minutes). For design purposes, the proportional gain of the PI block can be considered relatively small and the integral time constant can be set within $\tau_{I}=[10,20] \mathrm{s}$.

TABLE I

PARAMETERS OF THE CONTROLLER

\begin{tabular}{c|c|c|c}
\hline \multicolumn{2}{c|}{ PI control unit } & \multicolumn{2}{c}{ Corrective control unit } \\
\hline$K_{I}$ & $K_{P}$ & $T_{\text {delay }}$ & $N_{c}$ \\
\hline 0.01 & 0.1 & $10 \mathrm{~s}$ & 4 \\
\hline
\end{tabular}

As discussed before, $N_{C}$ indicates a trade-off between controller performance and computational expense since larger $N_{C}$ could translate to higher performance and higher computational burden. In this fashion, $N_{C}=4$ is intuitively selected in this study. In addition, it is also assumed that calculation time of controller is $2 \mathrm{~s}$.

The problem in (6) and (7) can be solved by using various optimization methods. In this study, a sequential equality constrained quadratic programming method in IMSL library written for Fortran programming language is adopted. In particular, we are using NNLPF function. It should be noted that simulation with $N_{C}=4$ control steps, on a system with 45 variables (22 DGs with active and reactive power control variables and 1 OLTC control variable) lasts $0.75 \mathrm{~s}$.

From an implementation point of view, with the specified delay and with estimated calculation time, it is possible to ensure control in the real time. One example of the hardware implementation is to have measurement stations at the every DG node which would wirelessly send data to a microcontroller plugged in computer (IoT devices). The computer can receive measured data through serial port and USB and process them to obtain the necessary control action.

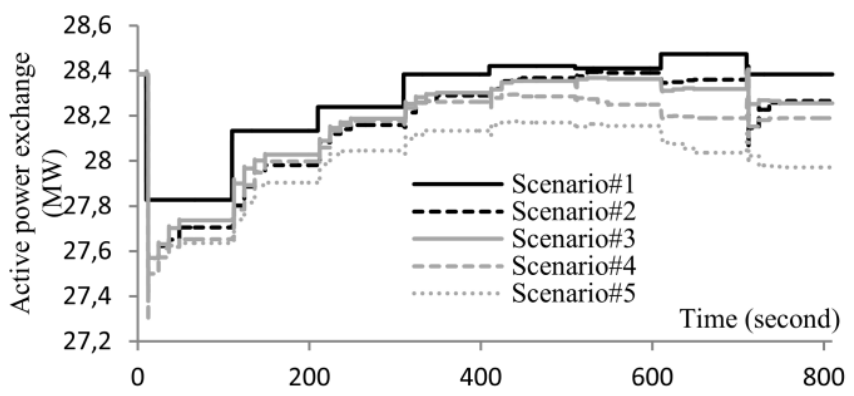

Fig. 10. Active power exchange inversely proportional to losses reduction.

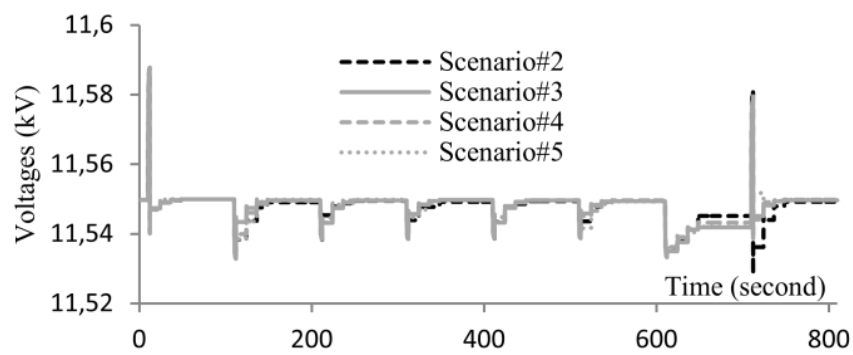

Fig. 11. Voltage at bus\#1166 with highest sensitivity on voltage violation.

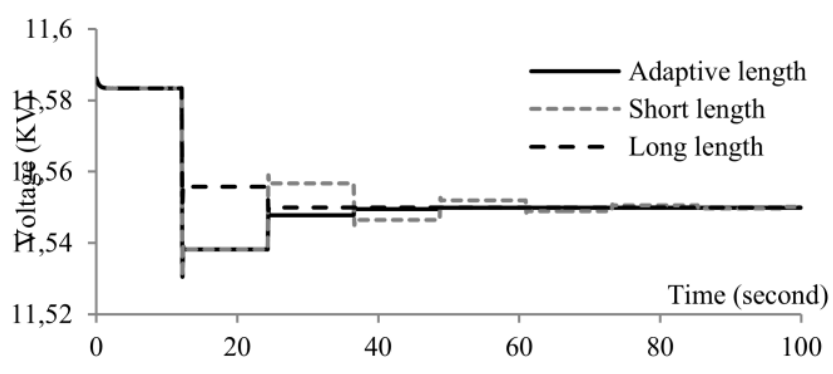

Fig. 12. Voltage at bus \#1166 with highest sensitivity on voltage violation.

In this work, is assumed that the computer "reacts" on the data send through UART or USB within milliseconds (which is enabled through the operating system scheduling thread). Thus, the real time operation is theoretically feasible. However, experimental implementation and verification is not within the scope of this paper.

\section{Losses Minimization Performance of the Controller}

\section{1) Impacts of the Overall Objective Function}

Fig. 8 shows response of the controller in fulfilling reactive power demand which is requested by TSOs. As discussed in part III-C-3, two strategies were proposed to improve performance of the controller. In each strategy, value of the slope, $\alpha \in\{1,0.1,0.05,0.01\}$, is simulated in turn. Examples of various $\alpha$ are compared with the original strategy (without penalty term in the objective function, $\alpha=0$ ), denoted as Baseline. It can be seen from Fig. 9 that in case of Baseline, performance of losses minimization can be comparative with the cases of two strategies. However, a lot of power fluctuation appears. This is because control variables requested by the controller move far from their actual values to attempt to minimize the losses. Unfortunately, such large 


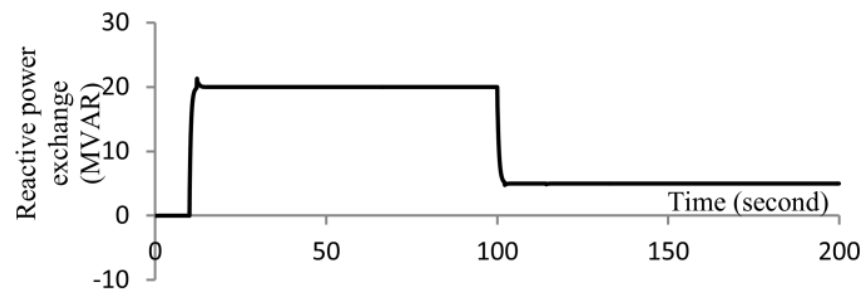

Fig. 13. Correction of reactive power exchange.

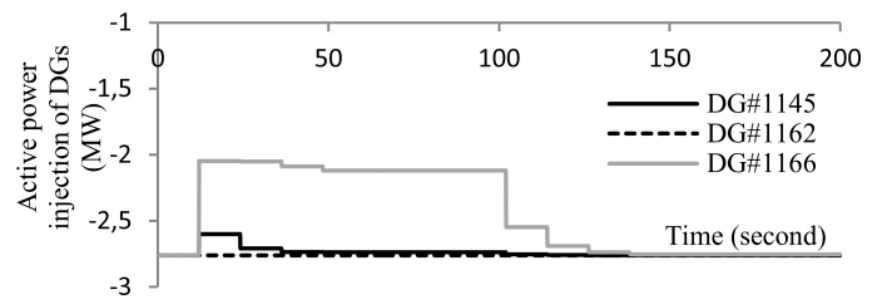

Fig. 14. Active power injection of DGs.

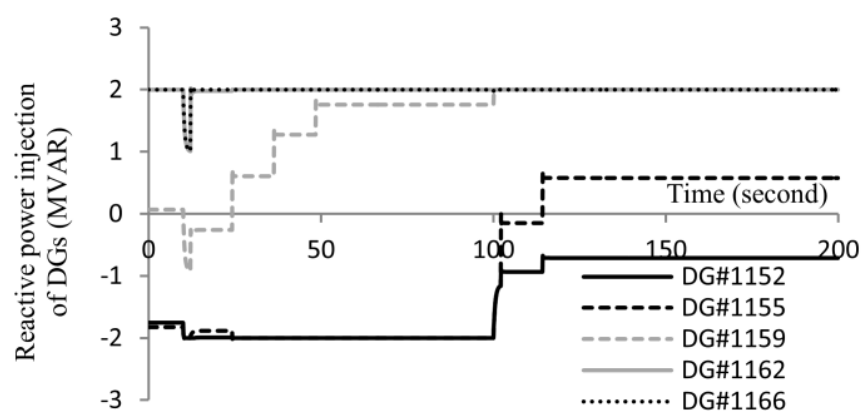

Fig. 15. Reactive power injection of DGs.

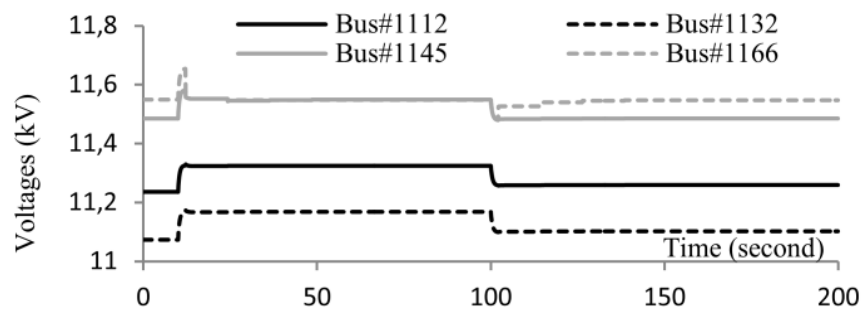

Fig. 16. Voltage at several buses.

change of control variables could aggravate inaccuracy of their sensitivities which are approximated by small perturbation around their actual values. By contrast, with introduction of two proposed strategies, the drawback of Baseline case can be solved.

In Strategy\#1, the controller displays significantly different performances with various slopes $\alpha$. On the other hand, introduction of a dead-band around actual value of each control variable in Strategy\#2 can alleviate the dependence between the controller performance and the slope $\alpha$, hence selection of $\alpha$ is simplified.

2) Impacts of Sensitivities Accuracy

This section shows that the performance of the controller is strongly dependent on the accuracy of the sensitivities. The

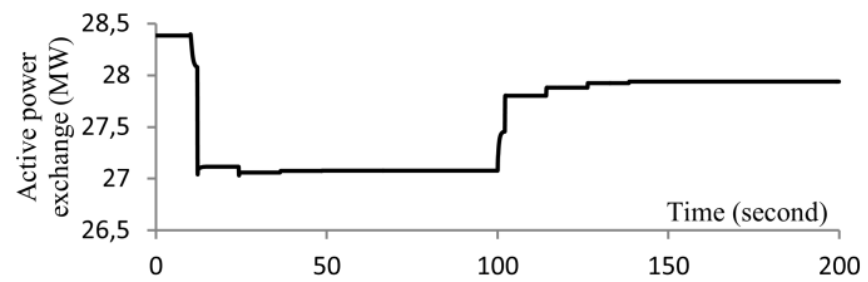

Fig. 17. Active power exchange inversely proportional to losses reduction.

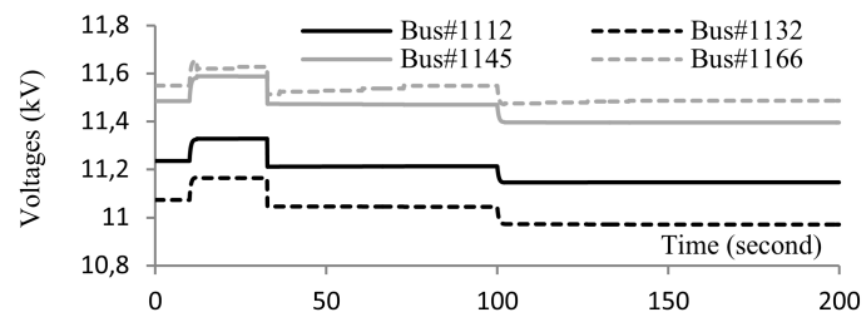

Fig. 18. Voltage of monitored buses.

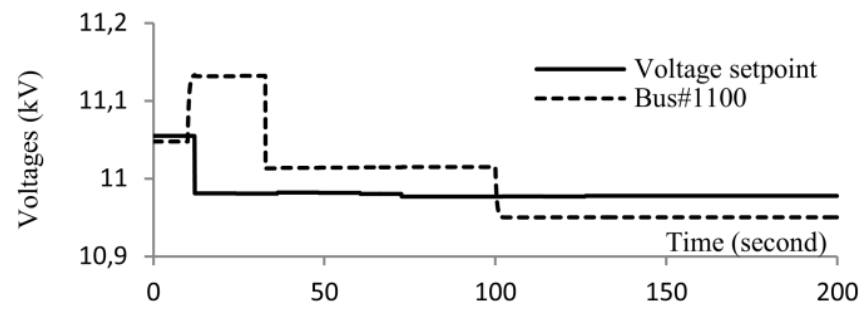

Fig. 19. Voltage at important points of OLTC transformer

performance of the controller was investigated in the following scenarios with the same operation conditions as in the previous section. The accuracy of the sensitivities decreases from Scenario\#2 to \#5.

- Scenario\#1: At each new operating point, optimal LF, calculated by a heuristic optimization algorithm, namely Mean Variance Mapping Optimization (MVMO) introduced in [12], is adopted in order to minimize the losses. This scenario apparently provides the best performance and hence, considered as a base case for comparison.

- Scenario\#2: The sensitivities are directly computed using LF calculation (not using the ANN) at each calculation step.

- Scenario\#3: The sensitivities are provided by the ANN with 66 input signals, $\left(v_{g}^{\text {meas }}, p_{g}^{\text {meas }}, q_{g}^{\text {meas }}\right)$ of all 22 DGs. This ANN is trained 3 minutes and 8 seconds on the CPU with RAM. After the training is measured mean squared error $7.01 .10^{-6}$.

- Scenario\#4: It is a repetition of Scenario\#3, however with only 33 input signals of 11 DGs. Since this is a smaller ANN than in the Scenario\#3, the training is shorter and it lasts $42 \mathrm{~s}$ using the same computer as previously mentioned. Mean squared error after the training is $3.55 .10^{-5}$. It should be noted that controller's performance decreases with reduction of ANN's input nodes (see Fig. 10).

- Scenario\#5: The sensitivities are initially calculated using $\mathrm{LF}$, and they are unchanged throughout simulation.

Fig. 10 shows that controller's performance in minimizing the losses is directly proportional to accuracy of sensitivities. Moreover, from Scenario\#3 and \#4 it is a further assertion that 


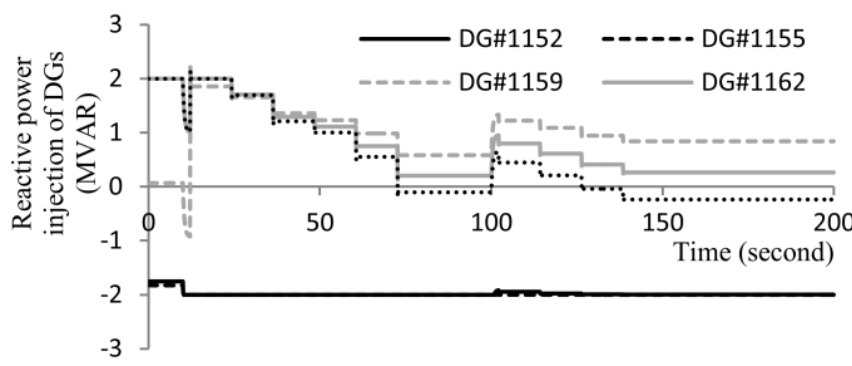

Fig. 20. Reactive power of DGs.

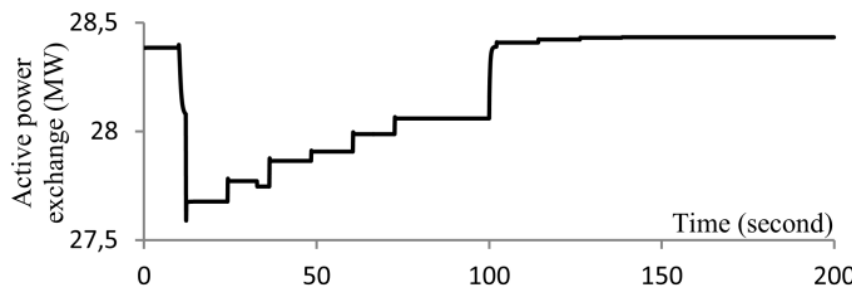

Fig. 21. Active power exchange as indication of loss reduction.

training performance of the ANN can be improved with increasing number of its inputs.

The proposed controller, carefully, but not conservatively reaching the targets (i.e., adaptive length of control horizon according to actual operation condition), as shown in Fig. 11, displays ability of regulating voltage subjected to inaccuracy of sensitivities.

\section{Voltage Correction Performance of the Controller}

\section{1) Impacts of Adaptive Selection of the Control Horizon}

In this scenario, it is assumed that the network operates at the condition with low load and high generation so that some buses are facing high voltage conditions.

To investigate performance of voltage regulation in terms of length of the control horizon in the proposed controller, three strategies such as adaptive length introduced in Part III-D, long one (e.g. $\left.N_{C}=2\right)$ and short one $\left(N_{C}=1\right.$, which is preferred by the network operator) are compared. In this investigation we assume that the CCU is triggered at $t=10 \mathrm{~s}$ merely for creating clearer pictures in Fig. 12. The voltage starts decreasing at $t=12 \mathrm{~s}$ when control actions apply. Controllers with the short and the adaptive prediction horizons have faster capacity in correcting the voltage than the one with a long horizon. As a result, at $t=22 \mathrm{~s}$, while the controller with short and the adaptive horizon manages to control losses, the controller with long horizon still continues correcting voltage. In other words, due to inaccurate model, controller with the short horizon produces excessive control actions, hence voltage breach at $t=24 \mathrm{~s}$. This is not repeated in the case with adaptive horizon, since in loss control mode the adaptive horizon becomes a long one, and controller calculates sequences of control actions to reach targets within several time steps ahead.

2) Voltage Correction without OLTC of the Transformer

Initially all DGs operate optimally to keep reactive power exchange at zero. At $t=10 \mathrm{~s}$ and $t=100 \mathrm{~s}$, a large amount of 20 MVAR power is exported and then down to 5 MVAR, respectively, is requested by TSOs. Fig. 13 presents response of the controller to fulfill this demand.

It can be seen from Fig. 14 and Fig. 15 that during the time interval between $t=10 \mathrm{~s}$ and $t=100 \mathrm{~s}$, when the large amount of reactive power is demanded, DGs closer to the substation try to fulfill reactive power exchange. Meanwhile other DGs at the end of several feeders, where voltages reach their limits $( \pm 5 \%$ nominal voltage), absorb reactive power to decrease voltage. But it can be seen that reactive power capacity of these DGs is insufficient to correct voltages. Drop of active power generation of DGs is needed, as shown in Fig. 14. As soon as the reactive power demand reduces to 5 MVAR at $t=100 \mathrm{~s}$, the CCU ensures that reactive power control brings the voltages into their limits, so active power curtailment should be eliminated. However, to avoid excessive control actions caused by inaccurate model or measurement noises, the CCU gradually ejects expensive control actions, thereby always keeping the voltages in the limits over the simulation time, as shown in Fig. 16.

At each triggered time $t=10 \mathrm{~s}$ and $t=100 \mathrm{~s}$, the $\mathrm{CCU}$ have four chances, defined with $N_{\text {step }}=4$, to reach its target as well as to improve its performance. Fig. 17 presents that with loss control mode the controller is able to improve its performance in minimizing the losses.

\section{3) Voltage Correction with OLTC of the Transformer}

With this scenario we aim at investigating voltage correction performance of the controller by using either DGs or OLTC of the transformer. Reactive power demand of TSOs is repetition of the last scenario, as shown in Fig. 13. It is noted that OLTC tap changer serves as a cheaper control action than active power curtailment control action, and that the time delay for the first tap change as well as for subsequent tap changes is $20 \mathrm{~s}$.

Fig. 18 presents voltage corrections by optimal control actions. The transformer takes one movement of tap-changer at $t=32 \mathrm{~s}$ to correct voltages. It can be seen from Fig. 19 that the voltage set-point ordered by the controller firstly changes at $t=12 \mathrm{~s}$, which pushes the OLTC monitored voltage at bus $\# 1100$ out of the acceptable dead-band. After the time delay of $20 \mathrm{~s}$, the movement of tap-changer is performed at $t=32 \mathrm{~s}$. It is worth emphasizing that at $t=24 \mathrm{~s}$, the $\mathrm{CCU}$ operates in the loss control mode with regulating reactive power of DGs only, as shown in Fig. 20. Later, since the decrease of reactive power exchange demand at $t=100 \mathrm{~s}$ does not cause voltage violation, the CCU is triggered just for the purpose of losses minimization.

It can be noticed from Fig. 17 and Fig. 21 that voltage correction using the control variables of the OLTC, instead of active power curtailment, leads to a higher minimization of the losses.

\section{CONCLUSIONS}

In this paper, a centralized control scheme based on a MPC and PI control unit has been successfully developed for accommodating the increasing integration of distributed generators into distribution networks and satisfying the different objectives of the operations by TSOs and DNOs. The 
proposed controller is based on sensitivities that replace the typical approach based on full LF calculation. The controller demonstrates to be effective for both voltage correction and losses minimization. The controller is only activated in predefined conditions. Thus, it helps significantly in relieving the computation burden. With adaptive MPC horizon length, the controller is able to quickly drive the network operating point close to the optimal solution, and without violation of the technical limits in each operation condition. From the perspective of the MPC practical implementation, the prediction horizon can be limited either by limiting a number of CPU computations (iterations) or by limiting the CPU time. Hence, for the tap change, the MPC can be designed and applied with a different time scale. Numerical results show that model inaccuracies or delays of the control actions do not affect the control actions. The implications (on controller's performance) of particular surfaces defining PQ constraints of the DGs and the PCC, depending on the activation and use of different control modes, and the corresponding specifications of the DGs, like in the study presented in [35], are part of future research.

\section{REFERENCES}

[1] ENTSO-E. Europe Power System 2040: Completing the Map \& Assessing the Cost of Non-Grid. Available online: https://tyndp.entsoe.eu/tyndp2018/power-system-2040/ (accessed on 12 April 2019).

[2] A. L'Abbate, G. Fulli, F. Starr and S.D. Peteves, "Distributed power generation in Europe: Technical issues for further integration," JRC Scientific and Technical Reports, 2007.

[3] K. Bell and S. Gill, "Delivering a highly distributed electricity system: Technical, regulatory and policy challenges," Energy Policy, Vol 113, pp. 765-777, Feb. 2018.

[4] European Commission, "COMMISSION REGULATION (EU) 2016/1388 Establishing a Network Code on Demand Connection”, Aug. 2016.

[5] M. Kurzidem, "Voltage support concept for the Swiss transmission system from 2011," Swissgrid, pp. 1-22, Ver. 1.4, 2011.

[6] L.N. Ochoa, F. Pilo, A. Keane, P. Cuffe and G. Pisano, "Embracing an adaptable, flexible posture: ensuring that future European distribution networks are ready for more active roles," IEEE Power Energy Mag., vol. 14, no. 5, pp. 16-28, Aug. 2016.

[7] J. Buire, F. Colas, J-Y Dieulot and X. Guillaud, "Stochastic optimization of PQ powers at the interface between distribution and transmission grids," Energies, 12(21):4057, Oct. 2019.

[8] A. Saint-Pierre and P. Mancarella, "Active Distribution System Management: A Dual-Horizon Scheduling Framework for DSO/TSO Interface under Uncertainty," IEEE Trans. Smart Grid, vol. 8, pp. 21862197, Sept. 2017.

[9] A. Keane, L.F. Ochoa, E. Vittal, C.J. Dent and G.P. Harrison, "Enhanced utilization of voltage control resources with distributed generation," IEEE Trans. Power Syst., vol. 26, no. 1, pp. 252-260, Feb. 2011.

[10] L.F. Ochoa, A. Keane and G.P. Harrison, "Minimizing the reactive support for distributed generation: enhanced passive operation and smart distribution networks," IEEE Trans. Power Syst., vol. 26, no. 4, pp. 2134-2142, Nov. 2011.

[11] G. Valverde and T. Van Cutsem, "Control of dispersed generation to regulate distribution and support transmission voltages," in Proc. IEEE PES PowerTech Conference, Jun. 2013.

[12] H.V. Pham, J.L. Rueda and I. Erlich, "Online optimal control of reactive sources in wind power plants," IEEE Trans. Sustainable Energy, vol. 5, no. 2, pp. 608-616, April 2014.

[13] S. Ali and F. Mutale, "Reactive power management at Transmission/Distribution interface," Proc. International Universities Power Engineering Conference, Sept. 2015.

[14] D.S. Stock, F. Sala, A. Berizzi and L. Hofmann, "Optimal Control of Wind Farms for Coordinated TSO-DSO Reactive Power Management," Energies, vol. 11, no. 173, Jan. 2018.
[15] G. Valverde and T. Van Cutsem, "Model Predictive Control of Voltages in Active Distribution Networks," IEEE Trans. Smart Grid, vol. 4, no. 4, pp. 2152-2161, Dec. 2013, doi: 10.1109/TSG.2013.2246199.

[16] J. Morin, F. Colas, S. Grenard, J-Y. Dieulot and X. Guillaud, "Coordinated predictive control in active distribution networks with HV/MV reactive power constraint," in Proc. IEEE PES Innovative Smart Grid Technologies Conference Europe, Oct. 2016.

[17] S. Li, T. Yu, T. Pu, J. Ming, and S. Fan, "Coordinated optimization control method of transmission and distribution network," in IEEE PES Asia-Pacific Power and Energy Engineering Conf., Oct. 2016, pp. 2215-2219.

[18] T. Ding, C. Li, C. Huang, Y. Yang, F. Li, and F. Blaabjerg, "A hierarchical modeling for reactive power optimization with joint transmission and distribution networks by curve fitting," IEEE Syst. J., vol. PP, no. 99, pp. 1-10, 2017.

[19] Z. Li, Q. Guo, H. Sun, J. Wang, and T. Xu, "A response-functionbased coordination method for transmission-distribution-coupled AC OPF," in IEEE PES Transm. Distrib. Conf. Exposition, 2018.

[20] X. Xing, J. Lin, C. Wan and Y. Song, "Model Predictive Control of LPC-Looped Active Distribution Network With High Penetration of Distributed Generation," IEEE Trans. Sustainable Energy, vol. 8, no. 3, pp. 1051-1063, July 2017, doi: 10.1109/TSTE.2016.2647259.

[21] N. Karthikeyan, J. R. Pillai, B. Bak-Jensen and J. W. Simpson-Porco, "Predictive Control of Flexible Resources for Demand Response in Active Distribution Networks," IEEE Trans. Power Systems, vol. 34, no. 4, pp. 2957-2969, July 2019, doi: 10.1109/TPWRS.2019.2898425.

[22] Y. Guo, Q. Wu, H. Gao, S. Huang, B. Zhou and C. Li, "Double-TimeScale Coordinated Voltage Control in Active Distribution Networks Based on MPC," IEEE Trans. Sustainable Energy, vol. 11, no. 1, pp. 294-303, Jan. 2020, doi: 10.1109/TSTE.2018.2890621.

[23] A. Pitì, G. Verticale, C. Rottondi, A. Capone and L. Lo Schiavo, "The role of smart meters in enabling real-time energy services for households: The Italian case," Energies, vol. 10, no. 199, Feb. 2017.

[24] T. Van Cutsem and C. Vournas, Voltage Stability of Electric Power Systems. Norwell, MA. USA: Kluwer, 1998.

[25] V.L. Paucar and M.J. Rider, "Reactive power pricing in deregulated electrical markets using a methodology based on the theory of marginal costs," in Proc. IEEE Large Engineering Systems Conference on Power Engineering, pp. 7-11, 2001.

[26] I. El-Samahy, C.A. Canizares, K. Bhattacharya and J. Pan, "An optimal reactive power dispatch model for deregulated electricity markets," in Proc. Power Engineering Society General Meeting, pp. 1-7, Jun. 2007.

[27] J. Zhong and K. Bhattacharya, "Toward a competitive market for reactive power," IEEE Trans. Power Syst., vol. 17, pp. 1206-1215, .Nov. 2002.

[28] Y. Zhang and Z. Ren, "Optimal reactive power dispatch considering costs of adjusting the control devices," IEEE Trans. Power Syst., Vol. 20, pp. 1349-1356, Aug. 2005.

[29] Rawlings, James Blake, David Q. Mayne, and Moritz Diehl. Model predictive control: theory, computation, and design. Vol. 2. Madison, WI: Nob Hill Publishing, 2017.

[30] T. Manrique, M. Fiacchini, T. Chambrion and G. Millerioux, "MPC tracking under time-varying polytopic constraints for real-time applications," in European Control Conference (ECC), Strasbourg, 2014, pp. 1480-1485, doi: 10.1109/ECC.2014.6862584.

[31] Ohta, Yuzo, et al. "Computer generated Lyapunov functions for a class of nonlinear systems." IEEE Transactions on Circuits and Systems I: Fundamental Theory and Applications 40.5 (1993): 343-354.

[32] Ohta, Yuzo. "On the construction of piecewise linear Lyapunov functions." Proceedings of the 40th IEEE Conference on Decision and Control (Cat. No. 01CH37228). Vol. 3. IEEE, 2001.

[33] United Kingdom Generic Distribution Network (UKGDS). [Online]. Available: http://sedg.ac.uk

[34] R. Singh, B.C. Pal and R.A. Jabr, "Statistical representation of distribution system loads using Gaussian mixture model," IEEE Trans. Power Syst., vol. 25, no. 1, pp. 29-37, Feb. 2010.

[35] A. Perilla, J. L. R. Torres, M. A. M. M. van der Meijden, A. Alefragkis and A. M. Lindefelt, "Analysis of a power factor regulation strategy for an embedded point-to-point MMC-HVDC system," 2018 IEEE International Energy Conference (ENERGYCON), Limassol, 2018, pp. $1-6$. 


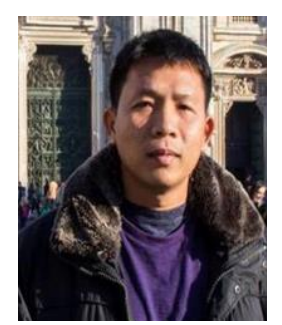

Hoa Minh Nguyen was born in Tra Vinh, Vietnam. $\mathrm{He}$ received his B.E. degree in electrical and electronics engineering and M.E. degree in control engineering from Ho Chi Minh City University of Technology, Vietnam in 2002 and 2005, respectively. He received the $\mathrm{Ph} . \mathrm{D}$. degree in Engineering and Applied Science from Department of Electrical Engineering and Computer Science, Idaho State University, USA. Dr. Hoa Minh Nguyen joined the faculty of Tra Vinh University, Vietnam in 2002, and became Dean, School of Engineering and Technology, Tra Vinh University, from 2006 to 2009 and from 2013 to 2019. He was appointed the vice rector, Tra Vinh University in 2019. His research interests include advanced control strategies, including sliding mode control, model predictive control, adaptive control, intelligent control, and their applications in renewable energy systems, power systems, and building energy management systems.

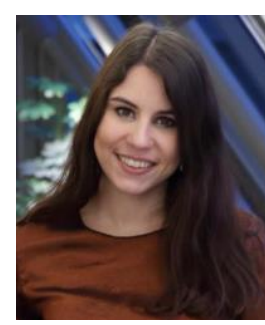

A. Lekić received the B.S., M.S., and Ph.D. degrees in electrical engineering from the School of Electrical Engineering, University of Belgrade, Serbia, in 2012, 2013, and 2017, respectively. Between 2012 and 2018 she has been a Teaching Assistant with the School of Electrical Engineering, University of Belgrade, and an Assistant Professor from 2018 to 2019. In 2019 she worked as a Postdoctoral Researcher at the Department of Electrical Engineering ESAT - ELECTA, KU Leuven and in the Institute EnergyVille, Genk, Belgium. From January 2020, Aleksandra works as an Assistant Professor at TU Delft, Faculty of Electrical Engineering, Mathematics and Computer Science in the group Intelligent Electrical Power Grids.

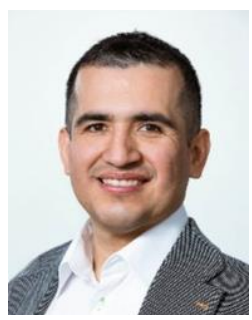

José Luis Rueda Torres was born in 1980 . He is currently an Associate Professor leading the research team on Stability, Control, and Optimization, within the Intelligent Electrical Power Grids Section, Department of Electrical Sustainable Energy, Delft University of Technology, Delft, The Netherlands. He received the Electrical Engineer Diploma from Escuela Politécnica Nacional, Quito, Ecuador, with cum laude honors in August 2004. From September 2003 till February 2005, he worked in Ecuador, in the fields of industrial control systems and electrical distribution networks operation and planning. In November 2009, he received the Ph.D. degree in electrical engineering from the National University of San Juan, obtaining the highest mark 'Sobresaliente' (Outstanding). Between August 2010 and February 2014 he worked as a postdoctoral research associate at EAN. His research interests include stability and control of power systems and multi-energy systems, power system operational planning \& reliability, and probabilistic and artificial intelligence methods. Currently, he is member of the Technical Committee on Power and Energy Systems of IFAC (International Federation of Automatic Control), Chairman of the IEEE PES Working Group on Modern Heuristic Optimization, Secretary of CIGRE JWG C4/C2.58/IEEE Evaluation of Voltage Stability Assessment Methodologies in Transmission Systems, Secretary of the IEEE PES Intelligent Systems Subcommittee, and Vice-Chair of the IFAC Technical Committee TC 6.3. Power and Energy Systems on Social Media.

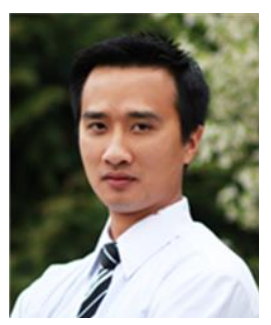

Hoan V. Pham was born in 1984. He received the B.E. from Can Tho University, Vietnam, in 2007, and the M.S. degree from Jeju National University, South Korea, in 2010, and the Ph.D. degree from the University of Duisburg-Essen, Germany, in 2015. $\mathrm{He}$ is currently a lecturer of electrical engineering with Tra Vinh University, Vietnam. Previously, he held the University of Strathclyde Postdoctoral Fellowship, Glasgow, U.K. His research interests include power system planning and optimizing operation, probabilistic and artificial intelligence methods, heuristic optimization and smart grids. 